\title{
Risk Factors and Prediction Model for Lateral Lymph Node Metastasis of Papillary Thyroid Carcinoma in Children and Adolescents
}

This article was published in the following Dove Press journal: Cancer Management and Research

\author{
Weili Liang $\mathbb{D}^{\prime}$ \\ Lei Sheng' \\ Liguang Zhou ${ }^{2}$ \\ Changyuan Ding' \\ Zhongyang Yao' \\ Chao Gao' \\ Qingdong Zeng' \\ Bo Chen (ID)'
}

'Department of Thyroid Surgery, General Surgery, Qilu Hospital of Shandong University, Jinan, 2500 I2, People's Republic of China; ${ }^{2}$ Department of Ultrasound, Shandong Provincial Hospital Affiliated to Shandong First Medical University, Jinan, People's Republic of China
Correspondence: Bo Chen

Department of Thyroid Surgery, General Surgery, Qilu Hospital of Shandong University, Wenhuaxi Road 107\#, Jinan, 2500 I2, People's Republic of China Tel +8618560085112

Email chenbo_78072I@hotmail.com
Purpose: Papillary thyroid carcinoma (PTC) in children and adolescents is prone to lateral lymph node metastasis (LNM), which is a high-risk factor for recurrence. However, few studies focused on identifying risk factors and establishing prediction models for lateral LNM of PTC in children and adolescents.

Patients and Methods: We retrospectively reviewed consecutive cases of children and adolescents with PTC undergoing thyroidectomy and cervical lymph node dissection between January 2009 and December 2019. The demographics and clinicopathologic features were collected and analyzed.

Results: A total of 102 children and adolescents with PTC were enrolled in our study; 51 of whom had lateral LNM (50\%). After adjusting for other risk factors, the independent risk factors for lateral LNM were multifocality (odds ratio [OR]: $6.04 ; 95 \%$ confidence interval $[\mathrm{CI}]: 1.653-22.092 ; \mathrm{p}=0.007)$, tumor size (OR: 1.752; 95\% CI: $1.043-2.945 ; \mathrm{p}=0.034$ ), and the number of central LNM (OR: 1.23; 95\% CI: 1.028-1.472; $\mathrm{p}=0.023$ ). The formula of the combined predictor is: Multifocality $+0.31 \times$ Tumor size $+0.115 \times$ Number of central LNM. The area under the receiver operating characteristic curve of multifocality, tumor size, number of central LNM, and the combined predictor was $0.706,0.762,0.748$, and 0.855 , respectively. When the value of the combined predictor was $\geq 2.2744$, lateral LNM could be predicted. The sensitivity and specificity of the predicted value were $82.4 \%$ and $74.5 \%$, respectively.

Conclusion: The independent risk factors for lateral LNM in children and adolescents with PTC were multifocality, tumor size, and the number of central LNM. The prediction model can better predict the presence of lateral LNM.

Keywords: papillary thyroid cancer, lymph node metastasis, risk factor, prediction model, pediatric surgery

\section{Introduction}

Although the incidence of thyroid nodules in children and adolescents is low, the rate of malignancy is higher compared with that recorded in adults. Papillary thyroid carcinoma (PTC) is the most common pediatric endocrine malignancy, accounting for $1 \%$ and up to $7 \%$ of all cancer cases in prepubertal children and adolescents, respectively. ${ }^{1}$ Compared to adults with PTC, children and adolescents with PTC have unique clinicopathological features, such as higher rates of lymph node metastasis (LNM), extrathyroidal extension (ETE), and pulmonary metastasis, and lower mortality rate. ${ }^{2,3}$ Even for pediatric patients with clinically node negative (cN0) PTC, the rate of LNM was as high as $75 \%{ }^{4}$ 
The presence, degree, and distribution of LNM have a significant impact on the recurrence rate in children and adolescents with PTC, ${ }^{5}$ ranging $10-35 \%{ }^{6,7}$ Sugino et $\mathrm{al}^{7}$ reported a $22.2 \%$ recurrence rate among 130 cases of PTC in children and adolescents, and identified the presence of clinically positive lymph node, ETE, and a number of metastatic lymph nodes $\geq 10$ as high-risk factors for recurrence. Byeon et al further proposed that lateral LNM rather than central LNM was an independent risk factor for disease recurrence. ${ }^{8}$ This finding was supported by other studies. ${ }^{5,9-11}$ Spinelli et al found that the rate of lateral compartment metastasis achieved $42.4 \%$ in children and adolescents with PTC. ${ }^{12}$

Thus far, there are no randomized trials investigating the treatment approaches for pediatric PTC; historically, the therapeutic approach is extrapolated from experience with adult patients. ${ }^{13,14}$ The American Thyroid Association guidelines only recommend therapeutic lateral lymph node dissection (LND) in patients with cN1b. However, they do not recommend preventive lateral LND in patients with $\mathrm{cN} 0$ or cN1a. In other words, lateral LND was performed only in pediatric patients in whom lateral LNM was suspected by preoperative imaging examination or intraoperative lymph node exploration. The diagnostic value of preoperative imaging examination, such as ultrasound for lateral LNM, remains questionable. $^{15,16}$ Based on an exceptional study in which all patients underwent therapeutic or prophylactic lateral LND, Ito et al reported that the sensitivity of preoperative ultrasound in the diagnosis of lateral LNM was as low as $27.3 \% .{ }^{15}$ Therefore, it is of great significance to investigate other methods for predicting lateral LNM, which may provide a beneficial supplement to imaging examination. The aim of this study was to identify risk factors for lateral LNM, establish a prediction model, and analyze its value.

\section{Patients and Methods}

\section{Patients}

Approval for this study was obtained from the ethics committee of Qilu Hospital of Shandong University in compliance with the tenets of the Declaration of Helsinki. Written informed consent and patient parental informed consent was obtained from all participants and their parents. Patient parental consent to review their medical records were required by the ethics committee. The data of consecutive children and adolescents with PTC undergoing surgery in our hospital between January 2009 and December 2019 were retrospectively analyzed. Children and adolescents were defined as those aged $\leq 21$ years according to the literature. ${ }^{13,14,17}$ All patients were diagnosed with high-resolution ultrasonography and, in most cases, disease was confirmed by fine-needle aspiration. Enhanced computed tomography was performed in each patient for a detailed preoperative evaluation of nodules and the cervical lymph nodes. All patients underwent thyroidectomy and cervical LND.

The exclusion criteria for patients were as follows: 1) lost to follow-up or with missing clinicopathological data; 2) presence of recurrent PTC; 3) presence of other pathologic types of thyroid cancer; 4) previous thyroid surgery or a history of irradiation involving the neck region; and 5) lack of lymph nodes in pathology despite undergoing LND.

Patient data were extracted from medical records and analyzed for various clinicopathologic factors, including demographics, tumor features, and postoperative pathologic details.

\section{Surgical Procedures}

Central LND was defined as a level of VI dissection, including the prelaryngeal, pretracheal, and unilateral or bilateral paratracheal lymph nodes. Lateral LND was defined as the excision of the lateral neck lymph nodes, including levels II-V; the minimum lateral LND range includes levels IIa, III, and IV. ${ }^{18,19}$ According to the American Thyroid Association guidelines for children with PTC, all patients underwent central LND regardless of suspected lymph nodes. ${ }^{20}$ Concerning the lateral lymph nodes, if the preoperative imaging examination indicated a suspected lymph node, we performed preoperative fine needle aspiration or intraoperative biopsy to reach a definitive diagnosis. Because the sensitivity and specificity of preoperative imaging examination were highly controversial, we performed lymph node biopsy based on the intraoperative exploration when the imaging examination did not indicate suspicious lymph nodes. Only patients with suspected or confirmed metastasis to the lateral neck region underwent lateral LND.

\section{Histopathologic Examination with Surgical Specimens}

Surgical specimens were microscopically examined by at least two experienced pathologists. Histopathologic examination included the cell sub-type of the main lesion, primary tumor size (measured as the longest diameter of 


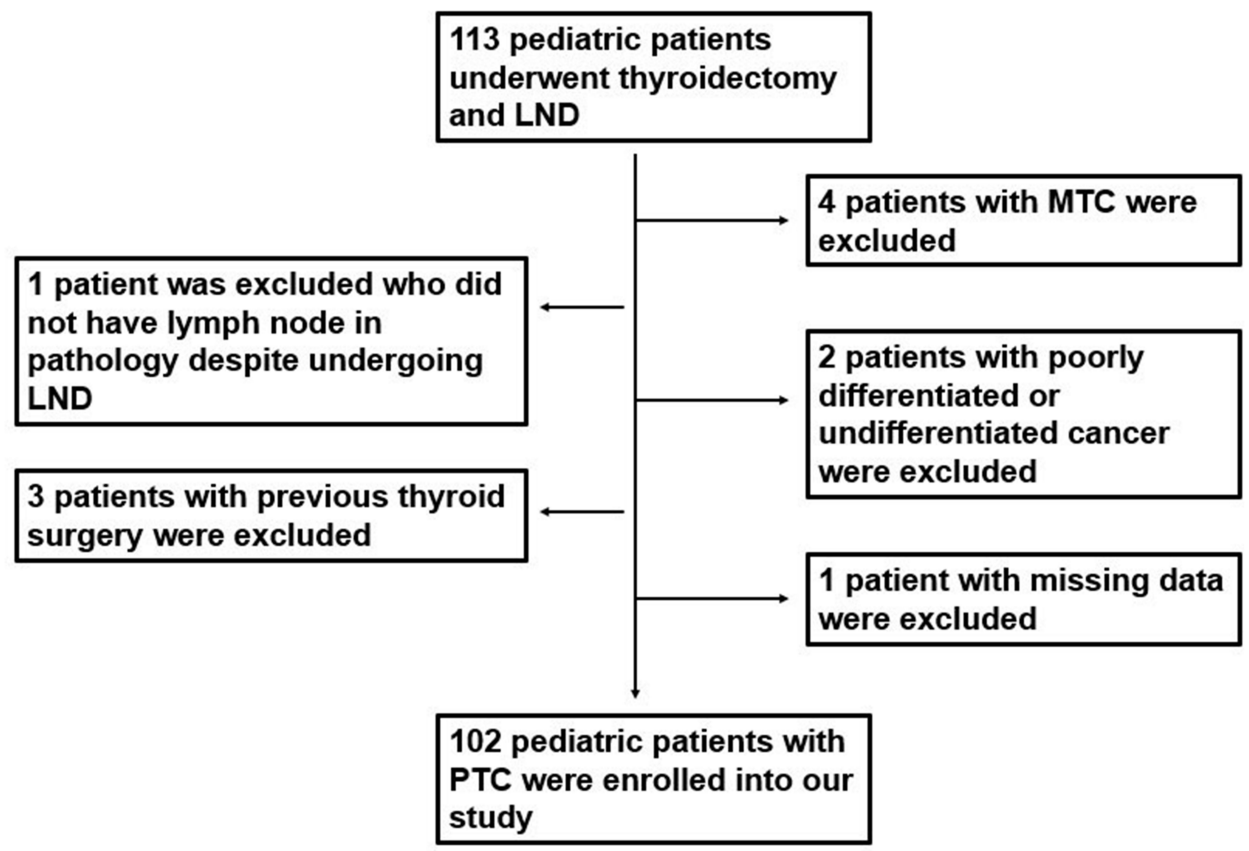

Figure I Flow chart of patient's selection.

Abbreviations: LND, lymph node dissection; MTC, medullary thyroid cancer.

the largest lesion), multifocality, ETE, lymphatic vascular (LV) invasion, intrathyroidal spreading, number of central LNM, and underlying conditions of the thyroid, such as Hashimoto's thyroiditis (HT). Multifocality was defined as having at least two lesions of PTC in a single lobe or two lobes. Intrathyroidal spreading referred to a major thyroid carcinoma with surrounding scattered small lesions, with features of heterotypic cells, psammoma bodies, and lymphatic vessel invasion. ${ }^{19}$ ETE was defined as tumor extension into the adjacent tissues and subdivided into minimal and massive ETE. Capsular invasion, equal to infiltration of the thyroid capsule, is a type of minimal ETE.$^{21}$ Herein, minimal ETE was defined as extension of the primary tumor to the perithyroidal soft tissues or the strap muscles. Massive ETE was defined as extension of the primary tumor to the trachea, esophagus, recurrent laryngeal nerve, larynx, subcutaneous soft tissue, skin, internal jugular vein, or carotid artery. ${ }^{22}$

\section{Statistical Analysis}

SPSS version 20.0 (IBM Corporation, Armonk, NY, USA) was used for data analysis, and $\mathrm{p}<0.05$ denoted statistical significance. The numerical variables were firstly tested for normality. Normally distributed variables were expressed as the mean \pm standard deviation and analyzed using $t$-tests. Non-normally distributed variables were expressed as the median with interquartile range and analyzed using the Kruskal-Wallis $H$-test. Categorical variables were compared using the $\chi^{2}$ test. Logistic regression was used for multivariate analysis. The prediction model is derived from the regression coefficient. A receiver operating characteristic (ROC) curve was plotted and the area under the curve was used to evaluate the diagnostic value of risk factors and the combined predictor. By calculating the Yoden index, the combined predictor corresponding to the maximum Yoden index was found, and its sensitivity and specificity were calculated.

\section{Results}

From January 2009 to December 2019, a total of 113 children and adolescents underwent surgery for thyroid cancer. Among them, 11 patients met the exclusion criteria and were not included in this study. The flow chart of patient selection is shown in Figure 1.

A total of 102 children and adolescents with PTC were enrolled into our retrospective study; of those, 76 (74.5\%) and $26(25.5 \%)$ were females and males, respectively. The median age was 17 years. Of the patients, 51 had lateral LNM (50\%). The baseline data of patients are shown in Table 1. 


\section{High Risk Factors for Lateral LNM of PTC in Children and Adolescents}

A univariate analysis showed that the groups of patients with and without lateral LNM were comparable in terms of sex $(p=0.173)$, HT $(p=0.336)$, intrathyroidal spreading $(p=0.135)$, and LV invasion $(p=0.461)$. There was significant difference between patients with and without lateral LNM in terms of age $(p=0.001)$, multifocality $(p<0.001)$, diffuse sclerosing variant (DSV)-PTC $(p=0.027)$, ETE $(\mathrm{p}=0.011)$, tumor size $(\mathrm{p}<0.001)$, and the number of central LNM $(\mathrm{p}<0.001)$ (Table 2$)$.

Table I The Baseline Data of 102 Pediatric Patients

\begin{tabular}{|c|c|}
\hline \multicolumn{2}{|l|}{ Clinicopathologic Features } \\
\hline Sex & \\
\hline Male/Female & $26 / 76$ \\
\hline Age (years) & \\
\hline Median with IQR (years) & $17(15-19)$ \\
\hline$\leq|5 /| 5-2 \mid$ & $31 / 7 \mid$ \\
\hline Tumor size & \\
\hline Median with IQR $(\mathrm{cm})$ & $2.1(1.2-3.0)$ \\
\hline$<2 \mathrm{~cm} / 2-4 \mathrm{~cm} />4 \mathrm{~cm}$ & $45 / 40 / 17$ \\
\hline Multifocality & \\
\hline Yes/No & $71 / 31$ \\
\hline DSV-PTC & \\
\hline Yes/No & $94 / 8$ \\
\hline ETE & \\
\hline No/minimal/massive & $44 / 46 / 12$ \\
\hline LV invasion & \\
\hline No/Yes & $94 / 8$ \\
\hline Intrathyroidal spreading & \\
\hline No/Yes & $82 / 20$ \\
\hline The number of positive central lymph nodes & 419 (Total) \\
\hline Median with IQR & $3(I-6)$ \\
\hline The number of total central lymph nodes & 721 (Total) \\
\hline Median with IQR & $5(4-10)$ \\
\hline The number of positive lateral lymph nodes & 416 (Total) \\
\hline Median with IQR & $6(3-12)$ \\
\hline The number of total lateral lymph nodes & I344 (total) \\
\hline Median with IQR & $21(14-34)$ \\
\hline
\end{tabular}

Note: Non-normally distributed variables were expressed as median with interquartile range (IQR).

Abbreviations: ETE, extrathyroidal extension; LV invasion, lymphatic vascular invasion; CLNM, central lymph nodes metastasis; DSV-PTC, diffuse sclerosing variant-papillary thyroid cancer; HT, Hashimoto's thyroiditis.
To exclude the influence of each variable factor, we subsequently performed a logistic regression analysis of lateral LNM. The independent risk factors for lateral LNM identified through this analysis were multifocality (odds ratio [OR]: 6.043 ; 95\% confidence interval [CI]: $1.653--$ $22.092 ; \mathrm{p}=0.007)$, tumor size (OR: 1.752 ; $95 \%$ CI: $1.043--$ $2.945 ; \mathrm{p}=0.034$ ), and the number of central LNM (OR: 1.23; 95\% CI: $1.028-1.472 ; \mathrm{p}=0.023)$. Age, DSV-PTC, and ETE were not independent risk factors for lateral LNM (Table 2).

\section{Establishment and Value of the Prediction Model}

According to the regression coefficient of the logistic regression analysis, the calculation formula of the prediction model is as follows:

Combined predictor $=$ Multifocality $+(0.561 / 1.799) \times$ Tumor size $+(0.207 / 1.799) \times$ Number of central LNM $=$ Multifocality $+0.31 \times$ Tumor size $+0.115 \times$ Number of central LNM.

We calculated the ROC curve of multifocality, tumor size, number of central LNM, and combined predictor (Figure 2). The area under the curve of the four predictors was $0.706,0.762,0.748$, and 0.855 , respectively; the combined predictor exhibited the greatest value.

Yoden index $=$ sensitivity + specificity -1

The maximum Yoden index was 0.569 , and the corresponding combined predictor was 2.2744 . Therefore, when the combined predictor was $\geq 2.2744$ (calculated using the above formula), the occurrence of lateral LNM could be predicted. The sensitivity and specificity of the predicted value were $82.4 \%$ and $74.5 \%$, respectively.

\section{Discussion}

Previous studies on the risk factors for lateral LNM have almost always targeted adults with PTC. These significant risk factors are mostly associated with tumor aggressiveness, which included central LNM, ETE, tumor multifocality, tumor size, LV invasion, etc. ${ }^{19,23,24}$ Currently, there is limited literature on the risk factors for lateral LNM of PTC in children and adolescents. Spinelli et al proposed that infiltration of the thyroid capsule, massive ETE, distant metastasis (DM), classic subtype of PTC, and central LNM were significantly associated with lateral LNM. ${ }^{12}$ Kim et al identified four tumor characteristics associated with an increased risk of LNM in pediatric PTC: larger tumor size; ETE; DM; and tumor 
Table 2 Risk Factors for Lateral LNM in Children and Adolescent Patients with PTC

\begin{tabular}{|c|c|c|c|c|c|c|c|c|c|}
\hline & & \multicolumn{2}{|c|}{ Lateral LNM } & \multirow[t]{2}{*}{$\mathbf{p}$} & \multirow[t]{2}{*}{ B } & \multirow[t]{2}{*}{ OR } & \multicolumn{2}{|l|}{ 95\% C.I. } & \multirow[t]{2}{*}{$\mathbf{P}$} \\
\hline & & No & Yes & & & & Lower Limit & Upper Limit & \\
\hline Age & $\begin{array}{l}\leq 15 \\
|6-2|\end{array}$ & $\begin{array}{l}8 \\
43\end{array}$ & $\begin{array}{l}23 \\
28\end{array}$ & 0.001 & $\begin{array}{l}0.770 \\
0\end{array}$ & $\begin{array}{l}2.16 \\
1\end{array}$ & 0.677 & 6.897 & 0.193 \\
\hline Sex & $\begin{array}{l}\text { Female } \\
\text { Male }\end{array}$ & $\begin{array}{l}41 \\
10\end{array}$ & $\begin{array}{l}35 \\
16\end{array}$ & 0.173 & & & & & \\
\hline Multifocality & $\begin{array}{l}\text { Yes } \\
\text { No }\end{array}$ & $\begin{array}{l}5 \\
46\end{array}$ & $\begin{array}{l}26 \\
25\end{array}$ & $<0.001$ & $\begin{array}{l}1.799 \\
0\end{array}$ & $\begin{array}{l}6.043 \\
1\end{array}$ & 1.653 & 22.092 & 0.007 \\
\hline DSV-PTC & $\begin{array}{l}\text { Yes } \\
\text { No }\end{array}$ & $\begin{array}{l}1 \\
50\end{array}$ & $\begin{array}{l}7 \\
44\end{array}$ & 0.027 & $\begin{array}{l}0.414 \\
0\end{array}$ & $\begin{array}{l}1.513 \\
1\end{array}$ & 0.122 & $18.7 \mid 4$ & 0.747 \\
\hline ETE & $\begin{array}{l}\text { Minimal ETE } \\
\text { Massive ETE } \\
\text { No }\end{array}$ & $\begin{array}{l}20 \\
3 \\
28\end{array}$ & $\begin{array}{l}26 \\
9 \\
16\end{array}$ & 0.029 & $\begin{array}{l}0.239 \\
0.403 \\
0\end{array}$ & $\begin{array}{l}1.270 \\
1.496 \\
1\end{array}$ & $\begin{array}{l}0.431 \\
0.183\end{array}$ & $\begin{array}{l}3.745 \\
12.227\end{array}$ & $\begin{array}{l}0.665 \\
0.707\end{array}$ \\
\hline LV invasion & $\begin{array}{l}\text { Yes } \\
\text { No }\end{array}$ & $\begin{array}{l}3 \\
48\end{array}$ & $\begin{array}{l}5 \\
46\end{array}$ & $0.46 I$ & & & & & \\
\hline HT & $\begin{array}{l}\text { Yes } \\
\text { No }\end{array}$ & $\begin{array}{l}9 \\
42\end{array}$ & $\begin{array}{l}13 \\
38\end{array}$ & 0.336 & & & & & \\
\hline Intrathyroidal spreading & $\begin{array}{l}\text { Yes } \\
\text { No }\end{array}$ & $\begin{array}{l}7 \\
44\end{array}$ & $\begin{array}{l}13 \\
38\end{array}$ & 0.135 & & & & & \\
\hline Tumor Size & & $1.5(1-2.5)$ & $3(2-4)$ & $<0.001$ & 0.561 & 1.752 & 1.043 & 2.945 & 0.034 \\
\hline The number of CLNM & & $3(1-4)$ & $5(3-7))$ & $<0.001$ & 0.207 & 1.230 & 1.028 & 1.472 & 0.023 \\
\hline
\end{tabular}

Notes: Variables in bold show statistical significance. Based on the regression coefficient from above logistic regression analysis, the formula for calculating prediction model of lateral LNM in children and adolescent patients with PTC can be obtained as follows: Combined predictor=Multifocality $+(0.56 \mathrm{I} / \mathrm{I} .799) \times$ Tumor size $+(0.207 / \mathrm{I} .799) \times$ The number of central LNM= Multifocality+ $0.31 \times$ Tumor size $+0.115 \times$ The number of central LNM.

Abbreviations: ETE, extrathyroidal extension; LV invasion, lymphatic vascular invasion; CLNM, central lymph nodes metastasis; DSV-PTC, diffuse sclerosing variantpapillary thyroid cancer; HT, Hashimoto's thyroiditis.

multifocality. ${ }^{25}$ In the study of children and adolescents with PTC in a post-Chernobyl exposed region, Fridman et al suggested that lateral LNM was associated with the age and sex of patients, tumor size, minimal ETE, solid architectonic, extensive desmoplasia in carcinoma, presence of psammoma bodies, extensive involvement of the thyroid, and the central lymph node ratio (LNR; number of metastatic lymph nodes/number of dissected nodes). ${ }^{5}$ According to the above literature, lateral LNM in children and adolescents with PTC is also mainly associated with factors representing tumor aggressiveness and progression.

In this study, tumor size was significantly associated with lateral LNM, consistent with the results reported in other studies. ${ }^{5,25}$ Shi et al suggested that the OR for lateral LNM continued to increase by size relative to a $1-10 \mathrm{~mm}$ baseline, and multi-stratification by size could provide more precise estimates of the likelihood of LNM in adult patients prior to surgery. ${ }^{26}$ The present study indicated that, as the tumor size increased by $1 \mathrm{~cm}$, the risk of lateral LNM increased by 1.752 -fold. The area under the ROC of the tumor size was 0.762 , indicating the good accuracy of the tumor size for the prediction of lateral LNM. More importantly, the tumor size can be determined preoperatively by ultrasound, allowing doctors to closely examine the risk of lateral LNM prior to surgery.

The number of central LNM was another independent risk factor for lateral LNM of PTC in children and adolescents; this finding was supported by other studies on pediatric patients. ${ }^{5,12}$ Generally, central lymph node is the initial site for LNM, followed by the lateral lymph nodes. We found that, as the number of central LNM increased by 1, the risk of lateral LNM increased by 1.23 -fold. The area under the ROC of the number of central LNM was 0.748 . We previously analyzed risk factors for lateral LNM in adult patients with papillary thyroid microcarcinoma. 


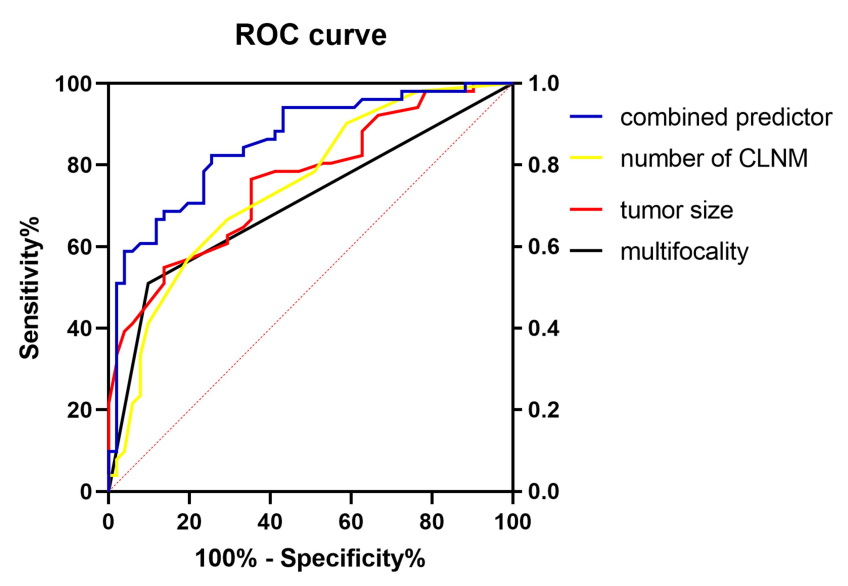

Figure 2 The ROC curve of multifocality, tumor size, number of central LNM and combined predictor. The area under the curve of the multifocality, tumor size, the number of CLNM and combined predictor was $0.706(95 \% \mathrm{Cl}, 0.603-0.808), 0.762$ (95\% Cl, 0.653-0.842), $0.748(95 \% \mathrm{Cl}, 0.670-0.853)$ and $0.855(95 \% \mathrm{Cl}, 0.782-$ 0.928), respectively (all $\mathrm{P}<0.05$ ).

Abbreviation: CLNM, central lymph node metastasis.

Although tumor size, ETE, intrathyroidal spreading, and tumor multifocality were associated with lateral LNM, only central LNM was a significant risk factor after adjusting for other risk factors. ${ }^{19}$

Some authors proposed that an increase in the number of tumors was associated with an increased risk of ETE, $\mathrm{LV}$ invasion, and $\mathrm{LNM}^{27}$ In pediatric patients with PTC, multifocality was also regarded as an independent risk factor for lateral $\mathrm{LNM}^{25}$ consistent with the results of the present study. We furthermore demonstrated that pediatric patients with multifocality had a 6.043-fold greater risk of lateral LNM than those without multifocality. The area under the ROC of the number of central LNM was 0.706 .

Regarding other influential factors, the sex of prepubertal children is not related to pediatric and adolescent PTC. However, after puberty, females are at a 4-5-fold higher risk of developing thyroid cancer than males. ${ }^{28}$ In this study, sex was not associated with lateral LNM of PTC in children and adolescents.

DSV-PTC is more frequently found in pediatric patients than adults. This type is associated with a more aggressive behavior and a less favorable outcome than conventional PTC. ${ }^{29}$ Although it was significant in univariate analysis, DSV-PTC was not an independent risk factor after adjusting for other risk factors in this study. This may be because the conventional subtype of PTC also has a high rate of lateral LNM in pediatric patients. ${ }^{12}$
Prepubertal children with DM are linked to poor clinical outcomes. ${ }^{16,23}$ The present study used a cut-off point of 15 years of age to classify the patients into prepubertal children and adolescents, according to previous literature. ${ }^{12}$ However, we did not observe a higher rate of lateral LNM in prepubertal children versus adolescents.

The presence of LV invasion, which was significantly associated with LNM in adult patients, should be considered as an indicator of aggressive clinicopathological features. ${ }^{23,30}$ In this study, LV invasion was not an independent risk factor for lateral LNM in pediatric PTC. This result is consistent with those of studies on lateral LNM of PTC in children and adolescents. ${ }^{12,25}$

ETE indicates poor prognosis for adult patients with PTC. Although ETE is often observed in PTC, its association with LNM remains controversial. In general, the presence of massive ETE (an adverse pathologic feature) is predictive of LNM. ${ }^{31}$ In pediatric patients with PTC, both massive ETE and infiltration of thyroid capsule were significantly associated with lateral LNM. ${ }^{12}$ In our study, ETE was not an independent risk factor for lateral LNM. This finding was consistent with those of our previous research on lateral LNM in adult patients with papillary thyroid microcarcinoma. ${ }^{19}$ This may be because the association of ETE with LNM was based on the tumor size. ${ }^{32}$

Thus far, there have been no reports on a prediction model for lateral LNM of PTC in children and adolescents. Central LNR (number of metastatic lymph nodes/number of dissected nodes) was adopted to predict lateral LNM. This may be a useful predictor for stratifying the likelihood of recurrence in pediatric patients undergoing central or lateral LND for pathologic N1a or N1b PTC. ${ }^{17}$ Fridman et al suggested that a high central LNR (eg, $>0.5$ ) was significantly associated with high risk for developing lateral LNM. ${ }^{5}$ However, LNR is not a prediction model which combines all independent risk factors by regression coefficient. Furthermore, LNR is susceptible to other factors. For example, a higher LNR is required to predict the presence of lateral LNM in patients with HT, and the central LNR ( $\mathrm{HT} \geq 0.4$; non-HT $\geq 0.6$ ) was independently associated with the occurrence of lateral LNM. $^{33}$ From this study, it can be concluded that the predictive value of the combined predictor (determined by calculating the area under ROC curve of multifocality, tumor size, number of central LNM, and combined predictor) is greater than that of any single risk factor. We further noted that a value for the combined predictor $\geq 2.2744$ was indicative of the occurrence of lateral 
LNM. The sensitivity and specificity values were $82.4 \%$ and $74.5 \%$, respectively, demonstrating the excellent predictive value of our model.

Firstly, to our knowledge, this is the first report of a prediction model for the development of lateral LNM of PTC in children and adolescents, in which multiple variables were systematically considered to estimate an individual risk of lateral LNM. Secondly, as this was not a prospective study, the surgical team was unable to perform preventive lateral LND, which may be the premise to ensure the accuracy of this study. However, we carefully conducted intraoperative exploration in cases without the presence of a suspicious lateral lymph node in preoperative imaging examination. We adopted the incision of thyroid surgery to perform a rapid pathological examination of enlarged lymph nodes in the III and IV sub-compartments, which could ensure the accuracy of the rate of lateral LNM observed in this study. Thirdly, this investigation included a larger sample. Because PTC is rare in children and adolescents, we collected data from a thyroid cancer treatment center over a 10 -year period. Finally, we performed statistical analysis of the numerical variables using the patient data. We did not group the tumor size and the number of central LNM into categorical variables as in previous studies. ${ }^{12,25}$ If a continuous numeric variable changed to a categorical variable through a cut-off point, the result may change as the cut-off point increases or decreases. ${ }^{23}$

Despite these advantages, this study had several limitations. Firstly, as retrospective research, the presence of potential selection bias in this study was inevitable. The low annual incidence of pediatric PTC poses challenges for the design of prospective randomized trials. This has led to the extrapolation of pediatric treatment approaches based on the literature concerning adults with PTC. Secondly, unfortunately, the number of central LNM can only be identified postoperatively by histopathologic examination. The other two predictors can be preoperatively detected through low-sensitivity neck ultrasonography. However, these factors identified postoperatively may be more suitable for guiding radioactive iodine therapy and accurate follow-up for pediatric patients. ${ }^{12}$

\section{Conclusion}

The independent risk factors for lateral LNM in children and adolescents with PTC were multifocality, larger tumor size, and higher numbers of central LNM. The calculation formula of the combined predictor for lateral LNM in pediatric PTC is as follows: Multifocality $+0.31 \times$
Tumor size $+0.115 \times$ Number of central LNM. The predictive value of these factors for lateral LNM is as follows (in descending order): the combined predictor, number of central LNM, tumor size, and multifocality. Therefore, the present model $(\geq 2.2744)$ showed excellent accuracy for predicting the occurrence of lateral LNM in pediatric patients with PTC.

\section{Abbreviations}

PTC, papillary thyroid cancer; LNM, lymph node metastasis; LND, lymph node dissection; cN0, clinically node-negative; cN1, clinically node-positive; ETE, extrathyroidal extension; ROC curve, receiver operating characteristic curve; HT, Hashimoto's thyroiditis; LV invasion, lymphatic vascular invasion; ATA, American Thyroid Association; DM, distant metastasis.

\section{Funding}

This study was supported by the Shandong Provincial Natural Science Foundation of China (Grant no. ZR2019PH082).

\section{Disclosure}

The authors report no conflicts of interest in this work.

\section{References}

1. Tracy ET, Roman SA. Current management of pediatric thyroid disease and differentiated thyroid cancer. Curr Opin Oncol. 2016;28 (1):37-42. doi:10.1097/CCO.0000000000000250

2. Francis GL, Waguespack SG, Bauer AJ, et al. Management guidelines for children with thyroid nodules and differentiated thyroid cancer. Thyroid. 2015;25(7):716-759. doi:10.1089/thy.2014.0460

3. Dzepina D. Surgical and pathological characteristics of papillary thyroid cancer in children and adolescents. Int $J$ Pediatr. 2012;2012:125389. doi:10.1155/2012/125389

4. Ngo DQ, Ngo QX, Van Le Q. Pediatric thyroid cancer: risk factors for central lymph node metastasis in patients with $\mathrm{cN} 0$ papillary carcinoma. Int $J$ Pediatr Otorhinolaryngol. 2020;133:110000. doi:10.1016/j.ijporl.2020.110000

5. Fridman M, Krasko O, Lam AK. Optimizing treatment for children and adolescents with papillary thyroid carcinoma in post-Chernobyl exposed region: the roles of lymph node dissections in the central and lateral neck compartments. Eur J Surg Oncol. 2018;44(6):733-743. doi:10.1016/j.ejso.2017.12.004

6. Feinmesser R, Lubin E, Segal K, Noyek A. Carcinoma of the thyroid in children-a review. J Pediatr Endocrinol Metab. 1997;10 (6):561-568. doi:10.1515/jpem.1997.10.6.561

7. Sugino K, Nagahama M, Kitagawa W, et al. Risk stratification of pediatric patients with differentiated thyroid cancer: is total thyroidectomy necessary for patients at any risk? Thyroid. 2020;30(4):548-556. doi:10.1089/thy.2019.0231

8. Byeon HK, Kim SB, Oh HS, et al. Clinical analysis of pediatric thyroid cancer: a single medical institution experience of 18 years. Ann Otol Rhinol Laryngol. 2019;128(12):1152-1157. doi:10.1177/ 0003489419868251 
9. Jeon MJ, Kim YN, Sung TY, et al. Practical initial risk stratification based on lymph node metastases in pediatric and adolescent differentiated thyroid cancer. Thyroid. 2018;28(2):193-200. doi:10.1089/ thy.2017.0214

10. Sapuppo G, Palermo F, Russo M, et al. Latero-cervical lymph node metastases $(\mathrm{N} 1 \mathrm{~b})$ represent an additional risk factor for papillary thyroid cancer outcome. $J$ Endocrinol Invest. 2017;40 (12):1355-1363. doi:10.1007/s40618-017-0714-y

11. Sung TY, Jeon MJ, Lee YH, et al. Initial and dynamic risk stratification of pediatric patients with differentiated thyroid cancer. $J$ Clin Endocrinol Metab. 2017;102(3):793-800. doi:10.1210/jc.2016-2666

12. Spinelli C, Tognetti F, Strambi S, Morganti R, Massimino M, Collini P. Cervical lymph node metastases of papillary thyroid carcinoma, in the central and lateral compartments, in children and adolescents: predictive factors. World J Surg. 2018;42(8):2444-2453. doi:10.1007/s00268-018-4487-z

13. Rubinstein JC, Herrick-Reynolds K, Dinauer C, et al. Recurrence and complications in pediatric and adolescent papillary thyroid cancer in a high-volume practice. J Surg Res. 2020;249:58-66. doi:10.1016/j. jss.2019.12.002

14. Palaniappan R, Krishnamurthy A, Rajaraman SS, Kumar RK. Management outcomes of pediatric and adolescent papillary thyroid cancers with a brief review of literature. Indian J Cancer. 2018;55 (1):105-110. doi:10.4103/ijc.IJC 48617

15. Ito $\mathrm{Y}$, Tomoda $\mathrm{C}$, Uruno $\mathrm{T}$, et al. Ultrasonographically and anatomopathologically detectable node metastases in the lateral compartment as indicators of worse relapse-free survival in patients with papillary thyroid carcinoma. World J Surg. 2005;29(7):917-920. doi:10.1007/ s00268-005-7789-X

16. Dou Y, Chen Y, Hu D, Xiong W, Xiao Q, Su X. Development and validation of web-based nomograms for predicting lateral lymph node metastasis in patients with papillary thyroid carcinoma. Gland Surg. 2020;9(2):172-182. doi:10.21037/gs.2020.01.11

17. Rubinstein JC, Dinauer C, Herrick-Reynolds K, Morotti R, Callender GG, Christison-Lagay ER. Lymph node ratio predicts recurrence in pediatric papillary thyroid cancer. J Pediatr Surg. 2019;54(1):129-132. doi:10.1016/j.jpedsurg.2018.10.010

18. Zhou L, Gao C, Li H, Liang W, Zeng Q, Chen B. Isthmic papillary thyroid carcinoma presents a unique pattern of central lymph node metastasis. Cancer Manag Res. 2020;12:3643-3650. doi:10.2147/ CMAR.S252692

19. Sheng LSJ, Han B, Lv B, et al. Predicting factors for central or lateral lymph node metastasis in conventional papillary thyroid microcarcinoma. Am J Surg. 2019. doi:10.1016/j.amjsurg.2019.11.032

20. American Academy of Pediatrics. Management guidelines for children with thyroid nodules and differentiated thyroid cancer. Pediatrics. 2018;142(6). doi:10.1542/peds.2018-3063

21. Jeon YW, Ahn YE, Chung WS, Choi HJ, Suh YJ. Radioactive iodine treatment for node negative papillary thyroid cancer with capsular invasion only: results of a large retrospective study. Asia Pac J Clin Oncol. 2016;12(1):e167-73. doi:10.1111/ajco.12159
22. Mete O, Rotstein L, Asa SL. Controversies in thyroid pathology: thyroid capsule invasion and extrathyroidal extension. Ann Surg Oncol. 2010;17(2):386-391. doi:10.1245/s10434-009-0832-7

23. So YK, Kim MJ, Kim S, Son YI. Lateral lymph node metastasis in papillary thyroid carcinoma: a systematic review and meta-analysis for prevalence, risk factors, and location. Int J Surg. 2018;50:94-103. doi:10.1016/j.ijsu.2017.12.029

24. Zhan S, Luo D, Ge W, Zhang B, Wang T. Clinicopathological predictors of occult lateral neck lymph node metastasis in papillary thyroid cancer: a meta-analysis. Head Neck. 2019;41(7):2441-2449. doi:10.1002/hed.25762

25. Kim J, Sun Z, Adam MA, et al. Predictors of nodal metastasis in pediatric differentiated thyroid cancer. J Pediatr Surg. 2017;52 (1):120-123. doi:10.1016/j.jpedsurg.2016.10.033

26. Shi RL, Qu N, Yang SW, et al. Tumor size interpretation for predicting cervical lymph node metastasis using a differentiated thyroid cancer risk model. Onco Targets Ther. 2016;9:5015-5022. doi:10.2147/OTT.S107187

27. Feng JW, Qu Z, Qin AC, Pan H, Ye J, Jiang Y. Significance of multifocality in papillary thyroid carcinoma. Eur J Surg Oncol. 2020;46(10):1820-1828. doi:10.1016/j.ejso.2020.06.015

28. Hogan AR, Zhuge Y, Perez EA, Koniaris LG, Lew JI, Sola JE. Pediatric thyroid carcinoma: incidence and outcomes in 1753 patients. J Surg Res. 2009;156(1):167-172. doi:10.1016/j. jss.2009.03.098

29. Kim SK, Park I, Woo JW, et al. Follicular and diffuse sclerosing variant papillary thyroid carcinomas as independent predictive factors of loco-regional recurrence: a comparison study using propensity score matching. Thyroid. 2016;26(8):1077-1084. doi:10.1089/ thy. 2016.0113

30. Sezer A, Celik M, Yilmaz Bulbul B, et al. Relationship between lymphovascular invasion and clinicopathological features of papillary thyroid carcinoma. Bosn J Basic Med Sci. 2017;17(2):144-151. doi:10.17305/bjbms.2017.1924

31. Kim JW, Roh JL, Gong G, et al. Extent of extrathyroidal extension as a significant predictor of nodal metastasis and extranodal extension in patients with papillary thyroid carcinoma. Ann Surg Oncol. 2017;24 (2):460-468. doi:10.1245/s10434-016-5594-4

32. Liu L, Oh C, Heo JH, et al. Clinical significance of extrathyroidal extension according to primary tumor size in papillary thyroid carcinoma. Eur J Surg Oncol. 2018;44(11):1754-1759. doi:10.1016/ j.ejso.2018.05.009

33. Zhu Y, Zheng K, Zhang H, et al. The clinicopathologic differences of central lymph node metastasis in predicting lateral lymph node metastasis and prognosis in papillary thyroid cancer associated with or without Hashimoto's thyroiditis. Tumour Biol. 2016;37 (6):8037-8045. doi:10.1007/s13277-015-4706-2

\section{Publish your work in this journal}

Cancer Management and Research is an international, peer-reviewed open access journal focusing on cancer research and the optimal use of preventative and integrated treatment interventions to achieve improved outcomes, enhanced survival and quality of life for the cancer patient.
The manuscript management system is completely online and includes a very quick and fair peer-review system, which is all easy to use. Visit http://www.dovepress.com/testimonials.php to read real quotes from published authors. 